\title{
Adaptive Image Enhancement Method ${ }^{*}$
}

\author{
Xizhen Han ${ }^{1}$, Jian Zhao ${ }^{1}$ \\ ${ }^{1}$ Changchun Institute of Optic, Fine Mechanics and Physics, Chinese Academy of Sciences, Changchun 130033, China \\ E-mail: xizhen_han@163.com, zhaojian6789@126.com
}

\begin{abstract}
The most important factor affecting the quality of image is the brightness and contrast. In order to improve the brightness and contrast of the image, this paper presents an adaptive image enhancement method. This method sets a threshold value according to the grayscale range of the image. If it is smaller than the threshold value, this paper uses auto gray levels enhancement. While if it is greater than the threshold value, this paper uses weighted gray levels equalization enhancement. Adaptive image enhancement method can not only enhance the low-contrast images, but also can enhance the dark images or the bright images. This method has a good robustness and the enhanced image has a better visual effect.

Index Terms - Adaptive image enhancement, Histogram equalization, Auto gray levels, weighted gray levels equalization.
\end{abstract}

\section{Introduction}

At present, the digital image processing technology is widely used in many fields of the national economy and national defense army building. Owing to the impacts of the image acquisition device itself and its working environment, the acquired images are often not clear enough in the dark area or the bright area. Or due to the bad weather such as fog, dust and haze, the contrast of the image is low and important details are not obvious. So the image enhancement processing has become a key link to the digital image processing field. Image enhancement is an important means to improve the image quality and the visual effect. And it provides good conditions for the following image processing and video tracking.

Image enhancement methods mainly include the spatial domain methods and the frequency domain methods. The spatial domain methods mainly include grayscale transformation method[1], histogram equalization, platform histogram equalization[2][3], laplace sharpening and so on. While the frequency domain methods mainly include homomorphic filtering method[4][5], wavelet transform $\operatorname{method}[6][7]$ and so on. It is discovered in the study on these enhancement methods that these methods have some limitations and they are not robust. Grayscale transformation method is simple, but easy to lose the details. Histogram equalization method is not good in enhancing the dark images or the bright images. It often appears saturated and overenhancement phenomenon. Platform histogram equalization is not generic method because its platform threshold is difficult to choose. Laplace sharpening can only enhance the image edges but can not enhance the brightness and the contrast. Homomorphic filtering can enhance the brightness of the image, but the contrast enhancement effect is not ideal. Wavelet transform is the same, and its calculation is very complex. Regarding for the limitations of the above methods, this paper presents an adaptive image enhancement method. The application prospect of adaptive image enhancement methods is very broad. It is widely used in engineering equipments, can not only greatly improve the image quality, but also improve the environmental adaptability of the device.

\section{Adaptive Image Enhancement Method}

In order to overcome the limitations of above image enhancement methods, this paper presents an adaptive image enhancement method, which can not only enhance the lowcontrast images, but also enhance the dark images or the bright images. And its calculation is simple. It can be used in the real-time image enhancement system.

Firstly, statistical the histogram of the image, and set an appropriate threshold based on grayscale range of the image. The gray level which is greater than $m$ is considered valid gray level. According to the valid gray levels, we can get the greatest gray level $k_{\max }$ and the smallest gray level $k_{\min }$. Secondly, when $k_{\max }-k_{\min }$ is less than the threshold value $k_{d}$, the image is considered as a low-contrast image. This paper uses auto gray levels enhancement. When $k_{\max }-k_{\min }$ is greater than the threshold value $k_{d}$, the image is considered as a bimodal image or a dark image or a bright image. This paper uses weighted gray levels equalization enhancement.

Auto gray levels enhancement can enhance the lowcontrast images well, and increase the range of the gray levels, without increasing the noise. The main idea of this method is that the grayscale range of the original image is mapped to the ideal grayscale range. The mapping function of the enhanced image is as follows:

$$
T_{1}(k)=\frac{k \times l}{k_{\text {max }}-k_{\text {min }}}-\frac{k_{\text {min }} \times l}{k_{\text {max }}-k_{\text {min }}}
$$

Where $k$ is a grayscale of the image, $l$ is the greatest grayscale. Suppose $r$ and $s$ respectively represent the original image and the enhanced image. In accordance with the grayscale mapping function, we can get the enhanced image:

$$
s(i, j)=T_{1}(r(i, j))
$$

Where $(i, j)$ means the $\mathrm{i}$ row and the $\mathrm{j}$ column.

\footnotetext{
* This work is supported by the program of academy-locality cooperation of the Chinese Academy of Sciences (2011CJT0004), the Jilin province science and technology development plan item (20090557 and 20125092).
} 
Weighted gray levels equalization enhancement is to revise the histogram of the image. Make the histogram remapping according to a certain rule, and then we get the new histogram. This method can enhance the dark image or the bright image, and won't produce saturation phenomenon and over-enhancement phenomenon. And this method also avoids to choose the threshold value just as the platform histogram equalization and dual-platform histogram equalization do. Suppose the total pixel number of the image is $n$, the number of the grayscale $k$ is $n_{k}$, the greatest grayscale is $l$. So the mapping function of the enhanced image is as follows:

$$
T_{2}(k)=\sum_{i=0}^{k} \frac{\left(\lambda_{1} n_{i}+\lambda_{2} n_{l-i}\right) \times l}{n}
$$

Where $k=0,1,2 \ldots l, \lambda_{1}, \lambda_{2}$ is the adjustment coefficients. In general, $\lambda_{1}>0.7, \lambda_{2}<0.3$. Suppose the original image is $u$, and the enhanced image is $f$, then we can get the formula:

$$
f(i, j)=T_{2}(u(i, j))
$$

\section{Experiments and Analysis}

In order to verify the effectiveness and the robustness of this adaptive image enhancement method, we experiment with three sets of images. Figure 1 is a low-contrast image. Figure 2 is a dark image. And figure 3 is a bright image. For each image, we use the histogram equalization method and this adaptive image enhancement method. The compared results are as follows. It is obvious according to the figures that histogram equalization makes figure 2 saturation, and makes figure 1 and figure 3 over-enhancement. While this adaptive image enhancement method can not only enhances the lowcontrast image, but also enhances the dark image and the bright image. This method makes the image details more clear without saturation and over-enhancement phenomenon.

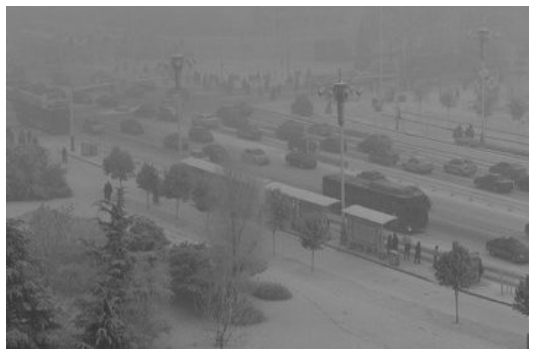

(a) original image

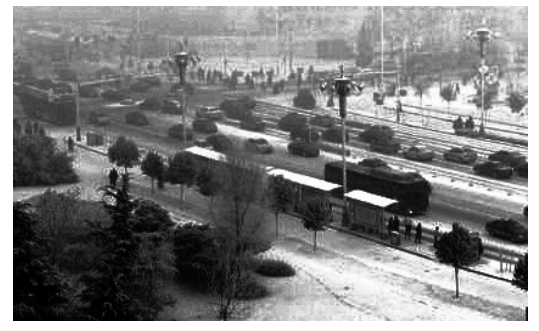

(b) histogram equalization image

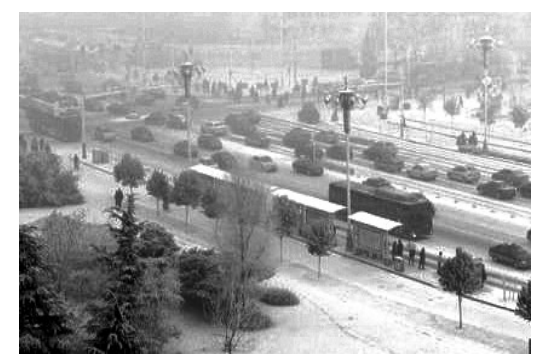

(c) this paper enhanced image

Fig.1 A Low-contrast Image

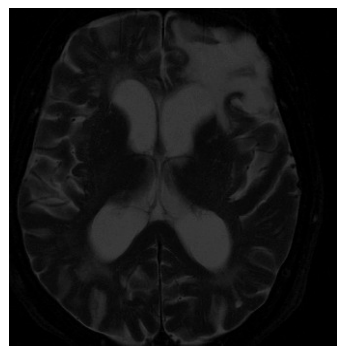

(a) original image

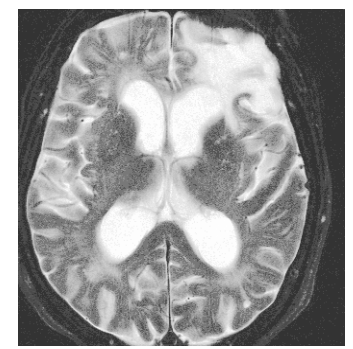

(b) histogram equalization image

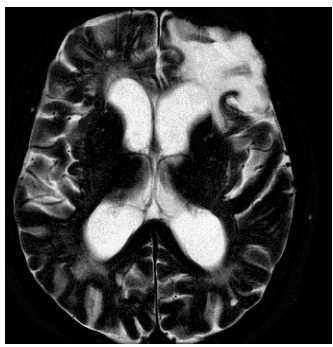

(c) this paper enhanced image

Fig.2 A Dark Image

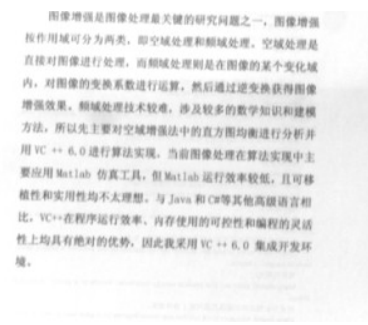

(a) original image

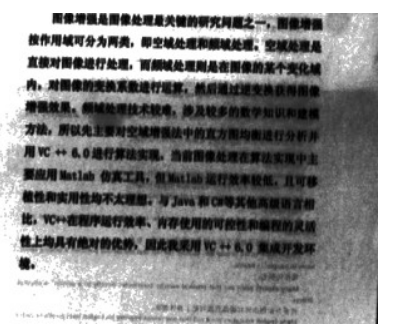

(b) histogram equalization image

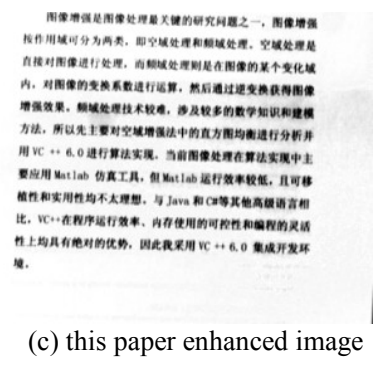

Fig. 3 A Bright Image

In evaluating the performance of various algorithms, we often combine the subjective evaluation with the objective evaluation. The subjective evaluation is to observe the details 
of the images by our eyes. In this paper, the objective evaluation uses Tenengrad standard, which can measure the detail information of the image[8]. The Tenengrad standard is defined as:

$$
T E N=\frac{1}{M N} \sum_{x} \sum_{y}|\nabla I| \quad \text { for }|\nabla I|>\mathrm{T}
$$

Where $T$ is the threshold value. TEN value is higher, the details of the image are richer, and the image quality is better. Now we calculate the TEN values of above three sets of images. The results are shown in Table 1.

TABLE I the compared result of $T E N$ values of above three sets of images

\begin{tabular}{|l|c|c|c|c|c|c|c|c|c|}
\hline & \multicolumn{3}{|c|}{ Fig.1 } & \multicolumn{3}{c|}{ Fig.2 } & \multicolumn{3}{c|}{ Fig.3 } \\
\cline { 2 - 9 } & (a) & (b) & (c) & (a) & (b) & (c) & (a) & (b) & (c) \\
\hline $\begin{array}{l}\text { TEN } \\
\text { values }\end{array}$ & 2.22 & 9.71 & 9.88 & 2.32 & 10.14 & 10.28 & 5.77 & 12.13 & 12.90 \\
\hline
\end{tabular}

We can see from Table 1 that histogram equalization and this adaptive image enhancement method both can greatly increase the $T E N$ value, but this paper method increases more than histogram equalization.

\section{Conclusion}

This adaptive image enhancement method can select different enhancement method by the grayscale range. When the contrast of the image is low, this paper uses auto gray levels enhancement, which can enhance the low-contrast image well, and can effectively suppress noise. When the brightness of the image is not uniform, this paper uses weighted gray levels equalization enhancement method, which can enhance the brightness and the contrast very well without saturation and over-enhancement phenomenon. This adaptive image enhancement method is simple, fast, and can be used in realtime video enhancement system.

\section{References}

[1] Daoqing Sheng, Genghuo Cheng, "The Research on the Image Enhancement Algorithm", Wuhan University of Science and Technology, 2011.

[2] Yiwei Mao, Guohua Gu, Qian Chen, Xiubao Sui, "Infrared Image Equalization Algorithm Based on Improved Plateaus Histogram", Optics \& Optoelectronic Technology, vol.10, no.5, pp.63-66, October 2012.

[3] Haijing Sun, Yanjie Wang, Weining Liu, "Enhancement of infrared image based on adaptive platform threshold and Laplace transformation", Chinese Optics, vol.4, no.5, pp.474-479, October 2011.

[4] Chunning Chen, Yanjie Wang, "Image contrast enhancement by homomorphic filtering in frequency field", Microcomputer Information, vol.23, no.2-3, 2007.

[5] Jun Xiao, Shoupeng Song, Lijuan Ding, "Research on the Fast Algorithm of Saptial Homomorphic Filtering", Journal of Image and Graphics, vol.13, no.12, pp.2302-2306, December 2008.

[6] Biao Chu, Gongqin Zhu, "Study on Image Denoising and Texture Classification Based on Wavelet Theory", Heifei: Hefei University of Technology, April 2008.

[7] Peng Jiang, Youxian Sun, "Study on Wavelet Application in Signal Denoise and Data Compression", Zhejiang: Zhejiang University, May 2004.

[8] Jinwei Zhou, Xiaolin Liu, "Research on Adaptive Local Image Enhancement Technique", Changsha: National University of Defense Technology, November, 2010. 